\title{
On the Uniqueness Theorem of Time-Harmonic Electromagnetic Fields
}

\section{Yongfeng Gui, Pei Li}

East China Research Institute of Electronic Engineering, Hefei, China.

Email: guiyongfeng80@163.com

Received November $25^{\text {th }}, 2010$; revised December $20^{\text {th }}, 2010$; accepted December $27^{\text {th }}, 2010$.

\begin{abstract}
The uniqueness theorem of time-harmonic electromagnetic fields, which is the theoretical basis of boundary value problem (BVP) of electromagnetic fields, is reviewed. So far there are many versions of the statements and proofs on the theorem. However, there exist some limitations and lack of strictness in these versions, for instance, the discussion of the uniqueness of solution without considering the existence of solution and the lack of strictness in the case of lossless medium. In contrast with the traditional statements and proofs, this paper introduces some important conclusions on operator equation from modern theory of partial differential equation (PDE) and attempts to solve the problems on the existence and uniqueness of the solution to operator equation which is derived from Maxwell's equations of time-harmonic electromagnetic fields. This method provides a novel and rigorous approach to discuss and solve the existence and uniqueness of the solution to time- harmonic fields in the new mathematical framework. Some important conclusions are presented.
\end{abstract}

Keywords: Time-Harmonic Fields, The Existence and Uniqueness of Solution, the Case of Lossless Medium, Operator Equation, Variational Principles, Weak Solution, Coercive Condition

\section{Introduction}

In the electromagnetics it has important significance to research time-harmonic electromagnetic fields. On one hand, most fields appeared in practical engineering problems have harmonic time variation and we can solve these engineering problems directly through researching time-harmonic fields. On the other hand, time-varying fields can be transformed into the superposition of time-harmonic fields with the Fourier series or Fourier transform $[1,2]$. Therefore, a thorough understanding and discussion on the existence and uniqueness of the solution to time-harmonic electromagnetic fields are important in the study of all electromagnetic fields and electromagnetic wave phenomena.

The necessity and importance of the uniqueness theorem consist in that if the appropriate initial-boundary value conditions of Maxwell's equations are given, then the solution is determined uniquely. So regardless of the method by which the equations are solved, the same solution will be obtained. Recently, the issue on the uniqueness has aroused much concern [3-9]. In this paper we mainly deal with the existence and uniqueness of the solution for time-harmonic electromagnetic fields. Firstly, this paper quotes some most influential versions of the uniqueness theorem of traditional time-harmonic electromagnetic fields theory and presents their proof method. Secondly, we point out the limitations and lack of strictness of traditional theory and make a result that the traditional theory has not yet solved the existence and uniqueness of the solution completely. Moreover, we cite the proof of existence and uniqueness of the weak solution to 0-Dirichlet problem of the Poisson equation as an example to indicate that it is a rigorous method adopting functional theory to discuss the existence and uniqueness of the solution to PDE. Finally, we introduce a novel consideration of the operator equation based on the modern theory of PDE, derive the operator equations of the time-harmonic electromagnetic fields from Maxwell equations, point out the substaintial difficulty in the process of proving the existence and uniqueness of the solutions and present some important conclusions.

\section{The Statements and Proof Method of Traditional Theory}

Maxwell's equations for time-harmonic fields are

$$
\nabla \times \vec{H}(\vec{r})=\vec{J}(\vec{r})+j \omega \vec{D}(\vec{r})
$$




$$
\begin{aligned}
& \nabla \times \vec{E}(\vec{r})=-j \omega \vec{B}(\vec{r}) \\
& \nabla \bullet \vec{D}(\vec{r})=\rho(\vec{r}) \\
& \nabla \bullet \vec{B}(\vec{r})=0
\end{aligned}
$$

The electric current continuity equation is

$$
\nabla \bullet \vec{J}(\vec{r})=-j \omega \rho(\vec{r})
$$

where $\vec{E}(\vec{r})$ is called the electric field intensity $(\mathrm{V} / \mathrm{m})$, $\vec{H}(\vec{r})$ the magnetic field intensity $(\mathrm{A} / \mathrm{m}), \vec{D}(\vec{r})$ the electric displacement or electric flux intensity $\left(\mathrm{C} / \mathrm{m}^{2}\right)$, $\vec{B}(\vec{r})$ the magnetic induction or magnetic flux intensity $\left(\mathrm{Wb} / \mathrm{m}^{2}\right), \vec{J}(\vec{r})$ the electric current density $\left(\mathrm{A} / \mathrm{m}^{2}\right)$ and $\rho(\vec{r})$ the electric charge density $\left(\mathrm{C} / \mathrm{m}^{3}\right)$.

There are many versions of the statements and proofs of the uniqueness theorem of time-harmonic fields in classical monographs and literatures. One of the most well known versions is cited as follows with the form of proposition.

Proposition 1. Uniqueness theorem of time-harmonic fields in simple connected domain

For the time-harmonic fields, considering a simply connected domain $V$ bounded by closed surface $S$, the medium is isotropic and linear, where both $V$ and $S$ contain only ordinary points. The solution satisfied Maxwell's Equations (1) and its boundary conditions must be unique if the following items are specified: (1) the sources density within $V$, that is, current density distribution $\bar{J}$ and equivalent magnetic current density distribution $\vec{J}_{m}$; and (2.1) the tangential components of the electric field or the tangential components of the magnetic field over whole boundary surface $S$ or (2.2) tangential electric field over part of the surface $S$ and tangential magnetic field over the remainder of $S$.

For the proof of Proposition 1, almost all the monographs and literatures adopt the method which belongs to a kind of "energy integrals" method [10]. Through constructing an expression of "energy integrals" based on Poynting theorem or Maxwell's equations, the "energy integrals" method has been applied to the proof of uniqueness theorem of the time-varying fields [11].

The proof of Proposition 1 under the assumption that the medium is lossy can be seen in [2,12-15]. For the case of a domain with complicated boundary, the entire domain can be divided into a number of sub-domain and make every sub-domain correspond with the simply boundary. So the multiply connected domain can be analyzed by decomposing it into the union of simply connected domain. The statements and proof can be seen in $[2,14]$.

It is assumed that medium is isotropic, linear and sources are located inside domain $V$ in Proposition 1. In fact, it can be generalized to the case of anisotropic medium and sources located outside domain $V$. For simplification, the isotropy and linear medium is discussed in this paper and the argument in linear anisotropic medium is similar.

\section{Deficiency of the Traditional Theory}

There exist some incompleteness in Proposition 1 and we explain it as follows.

\subsection{Existence of the Solution Has not been Proved Rigorously}

For the proof of uniqueness, traditional theory implies a physical judgment or premise that the solution must exist. It does not prove the existence of the solution and merely states that if a solution exists for given BVP then the solution is the only solution. However, it has been confirmed that the solution of many PDE do not exist really. For some equations if we assume the existence of the solution and construct the form of the difference solution, we can formally "obtain" the proof of the uniqueness. Obviously, the treatment is meaningless because the solution may not exist. For example, H. Lewy [16] provided an equation as follows:

$$
u_{x}+i u_{y}+2(i x-y) u_{t}=f(x, y, t) \quad \text { in } \Omega
$$

where $\Omega$ is a set satisfied $x^{2}+y^{2}<a,|t|<b, a$ and $b$ are arbitrary fixed positive number. Under the premise about existence of the solution of Equation (2) we can "obtain" the uniqueness of the solution by using reduction to absurdity. However, in fact there exists a function $f \in C^{\infty}(\Omega) \quad\left(C^{\infty}(\Omega)=\bigcap_{m=0}^{\infty} C^{m}(\Omega)\right.$ such that Equation (2) has no solution in $C^{1}(\Omega)$. Since $a$ and $b$ are arbitrary, Equation (2) has no solution in set

$$
\Sigma_{r}=\left\{(x, y, t) \in E^{3} \mid x^{2}+y^{2}+t^{2}<r^{2}\right\} \text { for all } r>0 .
$$

Detailed discussion of Equation (2) can be seen in [17].

In the sense of physics, the solution of practical electromagnetic BVP always exists. However, it does not mean that mathematical equations derived from the practical BVP must have a solution and the solution is unique. The existence of the solution still needs a rigorous mathematical proof and the judgment of physical concept is insufficient. Consequently, it is absolutely necessary and important to describe the reasonableness of mathematical model and prove the existence of the solution rigorously.

\subsection{The Lossless Case has not been Really Solved}

In the process of the traditional proof on Proposition 1, lossy medium is assumed, that is, at least one of conduction loss, polarization loss and magnetization loss is not 
equal to zero. The field in a lossless medium is treated as the limit of the corresponding field in a lossy medium when the dissipation approaches to zero. In sense of mathematics this treatment is not rigorous because the validation in the case of a parameter approaching zero does not guarantee the validation in the case of the parameter at the point of zero.

The proofs on the uniqueness theorem in lossless medium appeared in many books and literatures such as [2] [14] are only an interpretation based on the assumption that the case in lossless medium has been validated. The discussion on uniqueness theorem in lossless medium is avoided in [15], which write "The proof of the theorem hinges on the assumption that the permittivity and the permeability of the medium have a small imaginary part. Assume the medium is slightly lossy." Similarly, [18,19] have not made a definite conclusion and proof on the lossless case. Pozar in [20] considers that the solution for the lossless medium may be not unique unless the dissipation of medium is added. Hence, traditional theory has not given the proof of the uniqueness theorem in lossless medium strictly, which is a long-neglected problem. It is to be confirmed and proved whether there exists the uniqueness theorem in lossless medium.

For the final settlement of the problem this paper attempts to analyze the existence and uniqueness of the solution of time-harmonic fields by using related theory of functional analysis and PDE, offer a new kind of statement and proof method including considering of the existence.

\section{Theory and Application of Functional Analysis to PDE}

Time domain wave equation and frequency domain wave equation (Helmholtz equation) in electromagnetic fields belong to hyperbolic equation and elliptic equation, respectively. Obviously, PDE is a kind of operator equation. We will give a very famous example in which 0-Dirichlet BVP of Poisson equation is analyzed successfully to illustrate the application of functional analysis to the problem on the existence and uniqueness of the solution of operator equation.

Historically, the 0-Dirichlet BVP of Poisson equation $\nabla^{2} u=-f$ had been calculated directly for a long time. However, there exist great difficulties in proving the universality of the existence of the solution. After long-time endeavor, the idea is changed into the present method, that is, the weak solution of the equation is sought firstly, then its existence and uniqueness is proved, and finally its smoothness is determined. Thus, the following theorem is obtained.

Theorem 1. Consider the 0-Dirichlet BVP of Poisson equation

$$
\begin{aligned}
& \nabla^{2} u=-f \quad(\text { in } \Omega) \\
& \left.u\right|_{\partial \Omega}=0
\end{aligned}
$$

$\forall f \in L^{2}(\Omega)$, the equation must have a unique week solution, where $\Omega \subset R^{n}$ is a bounded open domain, $L^{2}(\Omega)$ represents quadratic integrable function space.

The definition of weak solution of Poisson equation (3.1), (3.2) is given in [21]. The Poincare inequality and Riesz representation theorem are used to prove the existence of weak solutions and reduction to absurdity is used to obtain the uniqueness of the solution [21]. Hence, the proof of Theorem 1, which is based on the theory of functional analysis, is rigorous.

Such mathematical method has become a basic research method in modern theory of PDE. As an indispensable tool in modern theory of PDE, functional analysis provides an important idea and model for solving the existence and uniqueness of time harmonic electromagnetic fields solutions.

For convenience of the discussion to operator equation, we cite some related definitions and important theorems in functional analysis ([21-25]) as follows.

Lemma 1. (Riesz representation theorem) For any bounded linear functional $f$ defined in a Hilbert space $H$, there exists unique $y_{f} \in H$ such that $f(x)=\left(x, y_{f}\right)$ and $\|f\|=\left\|y_{f}\right\|$ for every $x \in H$, where $\|\cdot\|$ represents a norm. Contrarily, for any $y_{f} \in H$, a bounded linear functional $f$ can be defined in terms of $f(x)=\left(x, y_{f}\right)$, and furthermore, $\|f\|=\left\|y_{f}\right\|$ holds (see [21,22,25]).

Riesz representation theorem indicates that a continuous linear functional can always be represented by an inner product.

Definition 1. Assume $a(u, v)$ is a bilinear functional defined in Hilbert space $H$,

1) $a(u, v)$ is called symmetrical if $\forall u, v \in H$ such that $a(u, v)=a(v, u) ; a(u, v)$ is called conjugate symmetrical if $\forall u, v \in H$ such that $a(u, v)=\overline{a(v, u)}$.

2) $a(u, v)$ is called bounded if $\exists M>0$ such that $|a(u, v)| \leq M \cdot\|u\| \cdot\|v\|(\forall u, v \in H)$.

3) $a(u, v)$ is called weakly coercive if $\exists \delta>0$ such that $|a(u, v)| \geq \delta\|u\|_{H}^{2}$ for any $u \in H$.

4) $a(u, v)$ is called coercive or positive definite if $\exists$ $\delta>0$ such that $a(u, v) \geq \delta\|u\|_{H}^{2}$ for any $u \in H$.

Definition 2. Assume $A$ is a linear operator defined in Hilbert space $X$,

1) $A$ is called self-conjugate or self-adjoint operator in $X$ if conjugate operator $A^{*}$ of $A$ exists and $A=A^{*}$.

2) $A$ is called symmetric operator in $X$ if $(A u, v)=(u, A v)$ for any $u, v \in X$.

3) $A$ is called weakly coercive operator in $X$ if $\exists$ $c>0$ such that $|(A u, u)| \geq c\|u\|^{2}$ for any $u \in X$.

4) $A$ is called positive definite operator in $X$ if $\exists$ $c>0$ such that $(A u, u) \geq c\|u\|^{2}$ for any $u \in X$. 
The above definitions still apply when $D(A)$ (the definition domain of operator $A$ ) is a dense linear subspace of $X$,

Lemma 2. (The Lax-Milgram theorem [22]) Assume $a(u, v)$ is a bounded, coercive and conjugate bilinear functional defined in a Hilbert space $H$, then there must exist a unique continuous linear operator $A \in L(H)$ such that $a(u, v)=(u, A v) \quad(\forall u, v \in H)$. Furthermore, $\left\|A^{-1}\right\|_{H} \leq 1 / \delta$, where $\delta$ is positive number.

The Lax-Milgram theorem indicates that the bilinear functional satisfied specific characteristic can constitute a linear operator with continuous inverse. The generalized Lax-Milgram theorem can be obtained when the coercive condition becomes the weak coercive condition [21].

\section{Modern Theory of Operator Equation in PDE}

The proof of the existence and uniqueness theorem of the solution to operator equation will be realized through the following steps.

\subsection{The Existence and Uniqueness of the Solution to Variational Equation as Well as Its Relation with Corresponding Variational Problem}

Theorem 2. Assume $U$ is a closed convex subset of real Hilbert space $H$. If $a(u, v)$ defined in $U$ is a bounded, coercive and bilinear functional, then for any $f \in H^{*}$ ( $H^{*}$ is a conjugate space of $H$, for Hilbert space $H^{*}=H$ ) there must exist unique $u \in U$ such that

$$
a(u, v)=(f, v) \quad(\forall v \in H)
$$

and $u$ is the solution of the following variational problem

$$
I(u)=\underset{v \in H}{\operatorname{Min}} I(v)=\underset{v \in H}{\operatorname{Min}}\left(\frac{1}{2} a(v, v)-(f, v)\right)
$$

Furthermore, the solution of variational problem is also unique (see [22,25-27]).

If the "bilinear functional" condition of Theorem 2 is changed into the "conjugate bilinear functional" condition, then the conclusions of Theorem 2 still hold for a complex Hilbert space, except that the expression of (4.2) is changed into the corresponding expression:

$$
I(u)=\operatorname{Min}_{v \in H} \frac{1}{2}(a(v, v)-(f, v)-(v, f)) .
$$

The coercive condition plays a very crucial role in the proof of Theorem 2 because it guarantees the existence and convergence of minimization sequence which is constructed in the proof. The completeness of space $U$ ensures that $u \in U$ is the limit of the sequence. Meanwhile, the symmetric condition guarantees that the minimum expression of functional has the meaning of existence.
If coercive condition in Theorem 2 is weakened into weak coercive condition, then the following theorem is obtained.

Theorem 3 ([27]). Assume $U$ is a closed convex subset of real Hilbert space $H$. If $a(u, v)$ defined in $U$ is a bounded, weak coercive and bilinear functional, then for any $f \in H^{*}$ there must exist unique $u \in U$ such that $a(u, v)=(f, v) \quad(\forall v \in H)$ and $u$ make the first order variation of functional $I(v)$ equal to zero, where $I(v)=1 / 2 a(v, v)-(f, v)$.

If the "bilinear functional" condition of Theorem 3 is changed into the "conjugate bilinear functional" condition, then the conclusions of Theorem 3 still hold for a complex Hilbert space, only the corresponding expression of variational problem becomes

$$
I(v)=1 / 2(a(v, v)-(f, v)-(v, f))
$$

\subsection{The Existence and Uniqueness of the Solution of Weak Form of Operator Equations as Well as Its Relation with Corresponding Variational Problems}

Theorem 2 and 3 have established the existence and uniqueness theorem of the solution to variational equations and indicated the only solution can be obtained by solving its corresponding variational problems. According to the relation between bilinear functional and linear operator, which can be found from Lemma 2, the existence and uniqueness theorem of the solution to weak form of operator equation can be obtained as follows.

Theorem 4. If $A$ defined in a Hilbert space $X$ is a linear, continuous and positive definite operator, then for any $f \in H^{*}$ there must exist unique $u \in X$ such that

$$
(A u, v)=(f, v) \quad(\forall v \in X)
$$

and $u$ is the solution of the following variational problem

$$
I(u)=\underset{v \in X}{\operatorname{Min}} I(v)=\underset{v \in X}{\operatorname{Min}}\left(\frac{1}{2}(A v, v)-(f, v)\right)
$$

Theorem 4 can be derived from Theorem 2 and its detailed proof can be seen in [22,25-27]. Equation (5.1) is called the weak form of operator equation $A u=f$ because compared with the original operator equation, Eq. (5.1) weaken the requirements to $u$. Theorem 4 can be generalized to the case of complex Hilbert space, except that the expression of corresponding variational problem becomes

$$
I(u)=\underset{v \in H}{\operatorname{Min}} \frac{1}{2}((A v, v)-(f, v)-(v, f))
$$

Clearly, positive definite operator is a stronger condition in the practical application. When the weak coercive condition of $A$ is satisfied, the following theorem will be 
obtained.

Theorem 5. If $A$ defined in a Hilbert space $X$ is a linear, continuous and weak coercive operator, then for any $f \in H^{*}$ there must be unique $x \in X$ such that

$$
(A x, v)=(f, v) \quad(\forall v \in X)
$$

and $x$ satisfied $\delta I(x)=0$, where

$$
I(x)=\frac{1}{2}((A x, x)-(f, x))
$$

that is, $x$ make the first order variation of functional $I(x)$ equal to zero [27].

The proof of Theorem 5 can be realized through taking advantage of the conclusion of Theorem 3. Theorem 5 can be generalized to the case of complex Hilbert space, except that the functional expression of corresponding variational problem becomes

$$
I(x)=\frac{1}{2}((A x, x)-(f, x)-(x, f))
$$

\subsection{The Existence and Uniqueness Theorem of Solution to Operator Equation}

Let $D(A)$ be a linear dense set in real Hilbert space $H$ and operator $A$ be mapping from $D(A)$ to $H$. For simplification, we assume the discussed equation $A u=f$ belongs to a kind of BVP of differential equation. At this time operator $A$ is a differential operator and domain $D(A)$ consists of smooth functions with certain differential order, which is greater or equal the order of differential operator. Therefore, the solutions of BVP on linear set $D(A)$ belong to the common sense solutions. If BVP have solutions on $D(A)$, then the solutions is called classical solution of BVP or its equivalent variational problems. However, in general we can not guarantee the existence of the solution to operator equation or variational problem. For BVP of Poisson equation in Theorem 1, if function $f$ has no continuity on boundary $\partial \Omega$, then the equation has no solution in linear set $D(A)=\left\{u \in C^{2}(\bar{\Omega}),\left.u\right|_{\partial \Omega}=0\right\}$. But, when $D(A)$ is extended to a linear set of Sobolev space

$$
H_{0}^{1}(\Omega)=\left\{v \in L^{2}(\Omega), D v \in L^{2}(\Omega),\left.v\right|_{\partial \Omega}=0\right\},
$$

at this time the weak form of the original equation on expended domain always has solutions. Obviously, this solution is not the solution of the original BVP in the sense of classical signification and is called a weak solution of the original equation [28-30]. We will build the connection between operator equation and variational problem through the weak form of operator equation and obtain the existence and uniqueness proposition of operator equation in the sense of weak solution.

Proposition 2 ([25]). Let $D(A)$ be a linear dense set in real Hilbert space $X$ and operator $A: D(A) \rightarrow X$. For operator equation

$$
A u=f(u \in D(A))
$$

where the definition domain of $A$ can be extended to space $H_{A}, H_{A}$ is a Hilbert space which is obtained by completion of $D(A)$ in terms of norm \|\|$_{A}$ and $\|u\|_{A}=\sqrt{(A u, u)}$. If $A$ is a linear, continuous, symmetric and positive definite operator, then the following conclusions hold.

1) for any $f \in X^{*}$ there must exist unique $u_{0} \in H_{A}$ such that

$$
\left(A u_{0}, v\right)=(f, v) \quad\left(\forall v \in H_{A}\right)
$$

and $u_{0}$ is the only solution of the following variational problem

$$
I(u)=\underset{v \in H_{A}}{\operatorname{Min}} I(v)=\underset{v \in H_{A}}{\operatorname{Min}}\left(\frac{1}{2}(A v, v)-(f, v)\right)
$$

2) If $u_{0} \in D(A)$, then $u_{0}$ is the classical solution of Equation (7.1). If $u_{0} \notin D(A)$, then $u_{0}$ is the weak solution of Equation (7.1).

Proof: It can be proved directly by using Theorem 4 and the definition of weak solution directly.

(7.2) corresponds to principle of virtual work and (7.3) corresponds to energy method.

If the conditions is weakened, the following proposition will be obtained.

Proposition 3([27]). Let $D(A)$ be a linear dense set in real Hilbert space $X$ and operator $A: D(A) \rightarrow X$. For operator equation

$$
A u=f \quad(u \in D(A))
$$

where the domain of $A$ can be extended to space $H_{A}$. If $A$ is a linear, continuous, symmetric and weak coercive operator, then the following conclusions hold.

1) for any $f \in X^{*}$ there must exist unique $u_{0} \in H_{A}$ such that

$$
\left(A u_{0}, v\right)=(f, v) \quad\left(\forall v \in H_{A}\right)
$$

and $u_{0}$ satisfied $\delta I\left(u_{0}\right)=0$, where

$$
I(u)=\frac{1}{2}(A u, u)-(f, u)
$$

2) If $u_{0} \in D(A)$, then $u_{0}$ is the classical solution of Equation (11.1). If $u_{0} \notin D(A)$, then $u_{0}$ is the weak solution of Equation (8.1).

Proposition 2 and 3 can be generalized to the case of complex Hilbert space, only need to change the functional expression of corresponding variational problem (7.3) and (8.3) into (5.3) and (6.3). In Proposition 2 and 3, symmetry condition guarantees that the values of functional expression must be real and makes the computation of extreme values feasible. The positive definite or weak 
coercive condition guarantees the existence of inverse operator and make the solution of weak form of operator equation exist.

Proposition 2 and 3 have summarized the existence and uniqueness theorems of weak solution to operator equation, moreover, provided a feasible and effective solving method of operator equation in the view of variational principle.

\section{The Application of Operator Equation Theory in the Time-Harmonic Fields}

Based on the statements above, we can get an idea on the proof of uniqueness theorem of time-harmonic electromagnetic fields. First we should deduce an operator equation (or weak form of Helmholtz equation and variational problem) from Maxwell's equation, then examine whether the operator is a linear, continuous, symmetric and positive definite (or weak coercive) operator, finally by means of Proposition 2 and 3 obtain a conclusion about it.

In the following we firstly prove the existence and uniqueness theorem of Poisson equation in the lossless case in brief, then deduce the weak form of Helmholtz equation and its variational problem, finally point out the substaintial issues appeared in the process of proving the existence and uniqueness of the solution to Helmholtz equation in the view of modern mathematical theory.

\subsection{The Realization on Proof of the Uniqueness Theorem of Poisson Equation}

For simplification we only discuss the scalar Poisson equation to show the application of Proposition 2 and 3. The argument in the case of vector Poisson equation is similar because vector Poisson equation can be decomposed into scalar Poisson equation.

Case A: For 0-Dirichlet BVP $\left(\left.u\right|_{\partial \Omega}=0\right)$ of the Poisson equation $\nabla^{2} u=-f \quad\left(u \in \Omega, f \in L^{2}(\Omega)\right)$, which is discussed in Theorem 1, Let $A=-\nabla \cdot \nabla$. By specifying the inner-product $(u, v)=\int_{\Omega} u v^{*} d \Omega$, we can verify that operator $A$ is linear, self-adjoint and positive definite. Hence, in accordance with Proposition 2, we know that both the solution of BVP of operator Equation (3) and the solution of the minimum value problem of the corresponding variational problem $J(u)=1 / 2(A u, u)-(f, u)$ exist uniquely and are equal. So within the region filled with the isotropic and uniform linear medium and under homogeneous boundary conditions, the weak solution of scalar Poisson equation must exist uniquely.

Case B: For Poisson equation with homogeneous and mixed boundary conditions

$$
\begin{aligned}
& -\nabla \cdot(\varepsilon \nabla u)=\rho \\
& \left.u\right|_{S_{1}}=0, \quad \varepsilon \frac{\partial u}{\partial n}+\left.\gamma u\right|_{S_{2}}=0
\end{aligned}
$$

where $S=S_{1} \cup S_{2}$ is the boundary surface. By specifying inner-product $(u, v)=\int_{\Omega} u v^{*} d \Omega$, we can easily verify that if $\varepsilon$ and $\gamma$ are non-negative and not equal to zero simultaneity, then operator $A=-\nabla \cdot(\varepsilon \nabla)$ is self-adjoint and positive definite. By Proposition 2 the unique weak solution must exist and it can be obtained by solving the minimum point of corresponding variational problem.

Case C: For Poisson Equation (9.1) with non-homogeneous boundary conditions, the boundary conditions is specified as follows:

$$
\left.u\right|_{S_{1}}=p, \quad \varepsilon \frac{\partial u}{\partial n}+\left.\gamma u\right|_{S_{2}}=q
$$

Through a transform $u^{\prime}=u-\phi$, we can get the new unknown function $u^{\prime}$, where $\phi$ is arbitrary function which satisfies non-homogeneous boundary conditions (10). Adopting the definition of inner-product $(u, v)=\int_{\Omega} u v^{*} d \Omega$, operator $A=-\nabla \cdot(\varepsilon \nabla)$ on variable $u^{\prime}$ becomes a self-adjoint operator and we can write the functional expression of $u$ by the functional expression of $u^{\prime}$. In terms of the discussion of segment B, we know that when $\varepsilon$ and $\gamma$ are real or real function, $A$ is a self-adjoint operator. So $J u$ can be written by the relation between the functional expression of $u$ and the functional expression of $u^{\prime}$. By the standard variational principle, the extreme point of $J u^{\prime}$ must exist uniquely. Hence, the extreme point of $J u$ must exist. When $\varepsilon$ and $\gamma$ are real and real function, the weak solution of Poisson equation with non-homogeneous boundary conditions must exist. Thus, we finally obtain the existence and uniqueness theorem of the solution to Poisson equation with non-homogeneous boundary conditions within region filled with the isotropic and uniform linear medium.

\subsection{The Application of Operator Equation Theory in Helmholtz Equation}

As a kind of elliptic PDE, the available conclusions in $[25,26,28,30,31]$ can not be applied to scalar wave equation. We will discuss vector wave equation of electric field by using of Proposition 2 and 3. The argument in the case of vector wave equation of magnetic field is similar. Scalar wave equation is a special case of vector wave equation.

\subsubsection{The Functional of Helmholtz Equation with Homogeneous Boundary Conditions in the Lossless Case}

By two curl Equations (1.1) and (1.2) of Maxwell's equa- 
tions we can deduce the double-curl equation of electric field as follows:

$$
\nabla \times\left(\frac{1}{\mu_{r}} \nabla \times \vec{E}\right)-k_{0}^{2} \varepsilon_{r} \vec{E}=-j \omega \mu_{0} \vec{J}
$$

As a vector problem, inner-product $(\vec{u}, \vec{v})=\int_{\Omega} \vec{u}^{*} d \Omega$ is specified. For the double-curl equation, let

$$
A=\nabla \times\left(\frac{1}{\mu_{r}} \nabla \times\right)-k_{0}^{2} \varepsilon_{r}
$$

And we have

$$
(A \vec{E}, \vec{F})=\int_{\Omega} \vec{F}^{*} \cdot\left[\nabla \times\left(\frac{1}{\mu_{r}} \nabla \times \vec{E}\right)-k_{0}^{2} \varepsilon_{r} \vec{E}\right] d \Omega
$$
into

By the second vector Green theorem, (12) is changed

$$
\begin{aligned}
(A \vec{E}, \vec{F}) & =\int_{\Omega} \vec{E} \cdot\left[\nabla \times\left(\frac{1}{\mu_{r}} \nabla \times \vec{F}^{*}\right)-k_{0}^{2} \varepsilon_{r} \vec{F}^{*}\right] d \Omega \\
& +\oint_{S} \frac{1}{\mu_{r}}\left[\vec{E} \times\left(\nabla \times \vec{F}^{*}\right)-\vec{F}^{*} \times(\nabla \times \vec{E})\right] \cdot \hat{n} d S
\end{aligned}
$$

If both $E$ and $F$ satisfy homogeneous Dirichlet boundary condition

$$
\hat{n} \times \vec{E}=0 \quad \text { on } S_{1}
$$

and homogeneous Neumann boundary condition

$$
\frac{1}{\mu_{r}} \hat{n} \times(\nabla \times \vec{E})+\gamma_{e} \hat{n} \times(\hat{n} \times \vec{E})=0 \quad \text { on } \quad S_{2}
$$

where $S=S_{1} \cup S_{2}$, then surface integral in (13) is equal to zero. If $\mu_{r}$ and $\gamma_{e}$ are real or real function, then (13) can be written into: $(A \vec{E}, \vec{F})=(\vec{E}, A \vec{F})$, that is, $A$ is a self-adjoint operator. Hence, the self-adjointness of $A$ defined by (11.2) need the following conditions: 1) $\varepsilon_{r}$, $\mu_{r}$ and $\gamma_{e}$ are real or real function; 2) boundary conditions are homogeneous. With these conditions substituting (11.2) (11.1) into (7.1) (5.3), we have

$$
\begin{aligned}
J \vec{E}= & \frac{1}{2} \int_{\Omega} \vec{E}^{*} \cdot\left[\nabla \times\left(\frac{1}{\mu_{r}} \nabla \times \vec{E}\right)-k_{0}^{2} \varepsilon_{r} \vec{E}\right] d \Omega \\
& +\frac{-j \omega \mu_{0}}{2} \int_{\Omega} \vec{E} \cdot \vec{J}^{*} d \Omega+\frac{j \omega \mu_{0}}{2} \int_{\Omega} \vec{J} \cdot \vec{E}^{*} d \Omega
\end{aligned}
$$

Assume that the medium is uniform and quote the first vector Green theorem and boundary conditions (14.1) and (14.2), (15) becomes

$$
J \vec{E}=\frac{1}{2} \int_{\Omega} \frac{1}{\mu_{r}}\left[(\nabla \times \vec{E}) \cdot\left(\nabla \times \vec{E}^{*}\right)-k_{0}^{2} \varepsilon_{r} \vec{E} \cdot \vec{E}^{*}\right] d \Omega
$$

$$
\begin{aligned}
& +\frac{j \omega \mu_{0}}{2} \int_{\Omega}\left(-\vec{E} \cdot \vec{J}^{*}+\vec{E} \cdot \vec{J}^{*}\right) d \Omega \\
& +\frac{1}{2} \int_{S_{2}} \gamma_{e}(\hat{n} \times \vec{E}) \cdot\left(\hat{n} \times \vec{E}^{*}\right) d S
\end{aligned}
$$

We can know that (11.1) corresponds to operator equation (7.1) or (8.1), (13) corresponds to weak form of operator Equation (7.2) or (8.2), (16) corresponds to the functional of variational problem (7.3) or (8.3). Meanwhile, the weak form of Helmholtz equation (13) is agreement with its corresponding variational problem $\operatorname{Min}(J \vec{E})$ or $\delta J \vec{E}=0$.

In the following we will discuss the property of the operator $A$. By definition we have

$$
(A \vec{E}, \vec{E})=\int_{\Omega} \vec{E}^{*} \cdot\left[\nabla \times\left(\frac{1}{\mu_{r}} \nabla \times \vec{E}\right)-k_{0}^{2} \varepsilon_{r} \vec{E}\right] d \Omega
$$

By the first vector Green theorem and boundary conditions (14.1) and (14.2), (17) becomes

$$
\begin{aligned}
(A \vec{E}, \vec{E}) & =\frac{1}{2} \int_{\Omega} \frac{1}{\mu_{r}}\left[(\nabla \times \vec{E}) \cdot\left(\nabla \times \vec{E}^{*}\right)-k_{0}^{2} \varepsilon_{r} \vec{E} \cdot \vec{E}^{*}\right] d \Omega \\
& -\oint_{S_{2}} \gamma_{e}(\hat{n} \times \vec{E}) \cdot\left(\hat{n} \times \vec{E}^{*}\right) d S
\end{aligned}
$$

By (18), we can not confirm that operator $A$ is positive definite or weak coercive because $(A \vec{E}, \vec{E})$ may be equal to zero. Because of the existence of term of $-k_{0}^{2} \varepsilon_{r} \vec{E} \cdot \vec{E}^{*}$ in integrand of (18), the Poincare inequality can not be used to prove the positive definite or weak coercive of the operator $A$. It is the substantial difficulty in the process of the application of Proposition 2 and 3. In mathematical, it belongs to one kind of eigenvalue problems, and its physical meaning represents resonance of electromagnetic fields. So this problem is an inherent property for time harmonic electromagnetic fields. For static fields no resonance can occur so the Poincare inequality can be used to prove the positive definite of Poisson operator. Therefore, for a concrete BVP of timeharmonic field if the frequency range is selected so as no resonance can occur, the operator in this frequency range will be positive definite or weak coercive. If the frequency is closed to resonance frequency of the structure to be analyzed, the operator will not be positive definite or weak coercive. For this situation if the operator equation is changed into linear algebraic equations in numerical algorithms we can find the matrix determinant is closed to zero or equal zero.

\subsubsection{The Further Discussion on the Solution to Helmholtz Equation}

Although the Lax-Milgram theorem plays a very important role in the solving of the weak solution and numeri- 
cal solution to PDE, the coercive or weak coercive condition of the theorem greatly limit its application scope. In [32] I. Babuska and A. K. Aziz generalized the LaxMilgram theorem under the weaker coercive condition, which greatly extends the application of the theorem. Furthermore, I. Babuska has also introduced another kind of coercive condition in [33], that is, strong Babuska condition, which further exert the application of the LaxMilgram theorem in finite element numerical method. However, It is to be determined whether strong Babuska condition of operator $A$ to Helmholtz equation is satisfied.

In the discussion of variational formula of FEM (finite element method [34]), some books and literatures think that the positive definite or the weak coercive conditions of operator is not necessary, only requiring that the operator is linear, continuous and symmetric, and the extremal solution of variation formula must be the solution of the original equation. Clearly, by Proposition 2 and 3 we can know that the above viewpoints are incorrect in the sense of modern mathematics theory. Proposition 2 and 3 have clearly indicated that the positive definite or the weak coercive conditions of operator is sufficient condition of the existence of $A^{-1}$, that is, sufficient condition of the existence of extreme value of functional.

\section{The Uniqueness of the Solution to Linear Algebraic Equations}

In terms of Proposition 2 and 3, operator equations can be solved with two kinds of distinct methods: variational method and the direct solving method of the weak form of operator equation. These numerical methods are eventually reduced to find the solution of linear algebraic equations, that is, to find the solution of matrix equations. Various methods of numerical solutions can be seen in [22, 35-42].

By Lax equivalence theorem (see [21]) and Lemma 2, we obtain that if the positive definite or the weak coercive conditions of corresponding operator are satisfied, then matrix determinant of linear algebraic equations obtained by the discretization of the operator equation is not equal to zero. For linear algebraic equations in which the number of unknown variables is equal to the number of equations, if matrix determinant of linear algebraic equations is not equal to zero, then the solution of linear algebraic equations must exist uniquely. Hence, the positive definite or the weak coercive conditions of operator guarantee the uniqueness of solution to matrix equation obtained by the discretization of the operator equation.

\section{Conclusions}

In this paper, the limitation and the lack of strictness on the uniqueness theorem of time-harmonic electromag- netic fields in traditional theory are pointed out; a new idea to solve the existence and uniqueness of the solution to time-harmonic fields' equations by means of the modern theory of PDE and functional analysis is described. The substantial difficulty is that the existence of term of $-k_{0}^{2} \varepsilon_{r} \vec{E} \cdot \vec{E}^{*}$ in integrand make the Poincare inequality not be used to prove the positive definite or weak coercive of the operator. The property of operator depends on whether resonance frequency of the structure to be analyzed belongs to the interested frequency range. The study work is being done and further results will be presented in future. Whether other mathematical method such as differentiable manifolds may be used to solve this problem is also interesting (private discussion with Dr. Q. Wang, 2006).

\section{Acknowledgements}

The authors wish to acknowledge Pro. Dou and Dr. Z. X. Wang of Southeast University for their constructive guidances and suggestions.

\section{REFERENCES}

[1] D. K. Cheng, "Linear System Analysis," Addison-Wesley Publishing Company, New Jersey, 1959.

[2] E. J. Rothwell and M. J. Cloud, "Electromagnetics," CRC Press, Boca Raton Florida, 2001. doi:10.1201/9781420058260

[3] Y. F. Gui, "A Rigorous and Completed Statement on Helmhotlz Theorem," Progress in Electromagnetics Research, Vol. 69, 2007, pp. 287-304.

doi:10.2528/PIER06123101

[4] L. X. Feng and F. M. Ma, "Uniqueness and Local Stability for the Inverse Scattering Problem of Determining the Cavity," Science in China Series A -Mathematics, Vol. 48, No. 8, 2005, pp. 1113-1123. doi:10.1360/022004-18

[5] W. Lin and Z. Yu, "Existence and Uniqueness of the Solutions in the SN, DN and CN Waveguide Theories," Journal of Electromagnetic Waves and Applications, Vol. 20, No. 2, 2006, pp. 237-247.

doi:10.1163/156939306775777297

[6] S. K. Mukerji, S. K. Goel, S. Bhooshan and K. P. Basu, "Electromagnetic Fields Theory of Electrical Machines-Part II: Uniqueness Theorem for Time-Varying Electromagnetic Fields in Hysteretic Media," International Journal of Electrical Engineering Education, Vol. 42, No. 2, 2005, pp. 203-208.

[7] D. Sjöberg, "On Uniqueness and Continuity for the Quasi-Linear, Bianisotropic Maxwell Equations, Using an Entropy Condition," Progress in Electromagnetics Research, Vol. 71, 2007, pp. 317-339. doi:10.2528/PIER07030804

[8] M. G. M. Hussain, "Transient Solution of Maxwell's Equations Based on Sumudu Transform," Progress in Electromagnetics Research, Vol. 74, 2007, pp. 273-289. 


\section{doi:10.2528/PIER07050904}

[9] X. L. Zhou, "On Independence, Completeness of Maxwell's Equations and Uniqueness Theorems in Electromagnetics," Progress in Electromagnetics Research, Vol. 64, 2006, pp. 117-134. doi:10.2528/PIER06061302

[10] R. Courant and D. Hilbert, "Methods of Mathematical Physics," Interscience Publishers, New York, 1962.

[11] J. A. Stratton, "Electromagnetic Theory," John Wiley \& Sons, New York, 1941.

[12] R. F. Harrington, "Time-Harmonic Electromagnetic Field," McGraw-Hill, New York, 1961

[13] A. Ishimaru, "Electromagnetic Wave Propagation, Radiation, and Scattering," Prentice Hall, London, 1991.

[14] K. Q. Zhang and D. J. Li, "Electromagnetic Theory for Microwaves and Optoelectronics," Electronics Industry Press, Beijing, 1994.

[15] J. A. Kong, "Electromagnetic Wave Theory," 2nd Edition, Wiley, New York, 1990

[16] H. Lewy, "An Example of a Smooth Linear Partial Differential Equation without Solution," Annals of Mathematics, Vol. 66, No. 1, 1957, pp. 155-158.

doi: $10.2307 / 1970121$

[17] M. Schechter, "Modern Methods in Partial Differential Equations: An Introduction," McGraw-Hill, Boston, 1977.

[18] W. C. Chew, "Waves and Fields in Inhomogenous Media," Van Nostrand Teinhold, New York, 1990.

[19] Y. Z. Lei, “Analytical Methods of Harmonic Electromagnetic Fields," Science Press, Beijing, 2000

[20] D. M. Pozar, "Microwave Engineering," 2nd Edition, Wiley, New York, 1998.

[21] G. Q. Zhang and Y. Q. Lin, "Functional Analysis," Peking University Press, Beijing, 1987.

[22] S. C. Brenner and L. R. Scott, "The Mathematical Theory of Finite Element Methods," Springer-Verlag, New York. 1996.

[23] K. Yosida, "Functional Analysis," 5nd Edition, Grun. der Math. Wissen., Springer-Verlag, New York, 1978.

[24] L. Liusternik, and V. Sobolev, "Elements of Functional Analysis," Frederick Ungar, New York, 1961.

[25] W. D. Lu, "The Variational Method for Differential Equation," Science Press, Beijing, 2003

[26] Z. C. Chen, "Partial Differential Equation," University of
Science and Technology of China Press, Heifei, 1993.

[27] Y. D. Wang, "The L2 Theory of Partial Differential Equation," Peking University Press, Beijing, 1989.

[28] Y. Z. Chen and L. C. Wu, "Two-Order Elliptic Equation and Elliptic Equations," Science Press, Beijing, 1997.

[29] R. A. Adams, "Sobolev Space," Academic Press, New York-San Francisco-London, 1975.

[30] D. Gilbarg and N. S. Trudinger, "Elliptic Partial Differential Equations of Second Order," Springer-Verlag, Heidelberg, New York, 1977.

[31] D. Kinderlehrer and G. Stampacchia, "Variational Inequalities and Its Application," Science Press, Beijing, 1991.

[32] I. Babuska and A. K. Aziz, "The Mathematical Foundations of the Finite Element Method with Application to Partial Differential Equations," Academic Press, New York, 1972.

[33] I. Babuska, "Analysis of Finite Element Methods for Second Order Boundary Value Problems Using Mesh Dependent Norms," Numerical Mathematics, Vol. 34, 1980, pp. 41-62. doi:10.1007/BF01463997

[34] A. Wexler, "Computation of Electromagnetic Fields," IEEE Transaction on MTT, Vol. MTT-17, 1969, pp. 416-439. doi:10.1109/TMTT.1969.1126993

[35] J. M. Jin, "The Finite-Element Method of Electromagnetism," Xi'dian University Press, Xi'an, 1998.

[36] O. C. Zienkiewicz and R. L. Taylor, "The Finite Element Method," 5th Edition, Stoneham, Butterworth-Heinemann, MA, 2000.

[37] K. W. Morton and D. F. Mayers, "Numerical Solution of Partial Differential Equations," Cambridge University Press, Cambridge, UK, 2005.

[38] M. N. O. Sadiku, "Numerical Techniques in Electromagnetics," CRC Press, Boca Raton, 1992.

[39] K. J. Bathe, "Finite Element Procedures," Prentice Hall, New Jersey, 1996.

[40] S. G. Mikhlin, "Variational Methods in Mathematical Physics," Macmillanm, New York, 1964.

[41] F. B. Hildebrand, "Methods of Applied Mathematics," 2nd Edition, Dover Publications, New York, 1992.

[42] R. E. Collin, "Foundations for Microwave Engineering," McGraw-Hill, New York, 1966. 